\title{
Benefits and Attributes of Plants \& Aromatherapy within A Healthcare Environment and Their Influence on Healthy \& Longevity
}

\begin{abstract}
Abbreviations: CAM: Complementary Alternative Medicine; CAT: Computerized axial-topography; ECG: Electrocardiographic; EEG: Electroencephalogram; EMG: Electromyogram; EPA: Environmental Protection Agency; EEA: European Environmental Agency; FTT: Fast Fourier transformations; HPA: Hypothalamopituitary-Adrenal; IAQ: Indoor Air Quality; NASA: National Aeronautics and Space Administration; SBS: Sick Building Syndrome; TCE: Trichloroethylene; VOC: Volatile Organic Compounds: WHO: World Health Organization
\end{abstract}

\section{Introduction}

Plants have been around since the beginning of time before humans existed. Plants are the lungs of the earth. They produce oxygen and make life possible. They provide precious moisture to the air, filter toxins, and regulate the climate. They influence the humidity, improve the air quality, bind dust, absorb carbon dioxide and other harmful substances and emit oxygen. During photosynthesis, all plants remove carbon dioxide and emit oxygen into the air for us to breathe. All plants remove impurities or volatile organic compounds from the air by absorbing them through their leaves.

Over the past several years' studies have shown how plants have positive healing effects on humans. In the last two million years, people have evolved in open spaces, clusters of vegetation, greenery and in nature, and recognize that plants profoundly affect their environment - their homes, workplaces, schools and healthcare facilities. Indoor air contaminants from benign sources such as furniture, drapes, carpets, insulation, paint and office machines can cause inflammation in humans. A variety of symptoms have been documented such as respiratory irritation, dizziness, headaches, skin rashes, nausea and vomiting. The Environmental Protection Agency reports that indoor air pollution poses serious acute and chronic health risks to individuals and that those health issues have an economic impact of tens of billions of dollars per year (EPA, 2000).

Indoor air contaminants from benign sources such as furniture, drapes, carpets, and insulation, paint and office machines can cause a variety of symptoms that include respiratory irritation, dizziness, headaches, skin rashes, nausea and vomiting. The EPA has issued a report stating that indoor air pollution may pose serious acute and chronic health risks with an economic impact of tens of billions of dollars per year. Research conducted by the National Aeronautics and Space Administration (NASA) shows that you may clean the air you breathe just by placing live plants throughout your home and office.

The National Aeronautics and Space Administration (NASA) showed that plants clean the air every time a person breathes just by placing household plants in the home office or indoor

Book Review
Lolume 8 Issue 2 - 2017
Lisa LaCova-Bhat*
Therident of Lmar Integrative Holistic Medicine \& Anti-Aging
*Corresponding author: Lisa LaCova-Bhat, President of
Lmar Integrative Holistic Medicine \& Anti-Aging Therapies,
Inc, USA, Email: lisa.lacova@gmail.com
Received: May 13, 2017 | Published: August 01, 2017

environments. NASA Scientists have studied and found that leaves, roots, soil, and microorganisms of plants have been evaluated in reducing indoor air pollutants such as cigarette smoke, organic solvents, and radon. (Walsh, 1984) Plant roots and microorganism destroy the pathogenic viruses, bacteria, organic chemicals, and pathogenic microorganisms, converting the air pollutants into a new plant tissue.

In recent research, Professor Roger Ulrich from Texas A\&M University focused on the effects of plants in hospitals. He found that plants improve the patient recovery time, reduce dependency on serious postoperative drugs, improve the effect of relaxation, and create a positive, holistic environment. Hospitals are slowly increasing in providing access to green spaces, gardens, and greenery, which have been proven to reduce stress, decrease the heart rate, reduce blood pressure and improve the patient satisfaction for patients, families, and staff. Even viewing nature, trees and pictures of nature has been shown to reduce hospital length of stay and result in fewer medications for patients. "Intuitively-based beliefs that exposure to trees, water and other nature [all elements] tend to foster psychological well-being... date as far back as the earliest large cities [1].

In addition, Urlich determined the creation of a healing environment has the propensity to affect anxiety, blood pressure, postoperative recovery, dose needed of analgesic medication and the duration of time a person stays in the hospital [1].

This study can be beneficial to the larger community because the development of a consistent healing environment in many different types of healthcare facilities such as TCM Clinics, hospitals, healthcare offices, integrative medical centers that can provide patients' continuity of care by means of a therapeutic environment, which could contain plants. Therapeutic environments that employ the use of plants can contribute to the overall improvement of health and of longevity within local communities. 


\section{Background}

The World Health Organization has estimated that $80 \%$ of the world's population relies solely or largely on traditional remedies for heath care and about two billion people rely heavily on medicinal plants.

Researchers have done studies that have shown how plants have a positive effect on humans. In the last 2 million years, people have evolved in open spaces, clusters of vegetation, greenery and in nature. People are starting to recognize how plants have a profound effect on our environment in our homes, workplaces, schools and hospitals. Professor Roger Ulrich from Texas A\&M University focused on the effects of plants in hospitals in areas such as in improving patient recovery time, reducing dependency on serious post operative drugs, on relaxation, and on creating a positive working environment for the on staff, when green plants were present. Healing environments have a major effect on anxiety; blood pressure, postoperative recovery, analgesic medication and the length of the hospital stay [1]. Part of complementary medicine, aromatherapy uses essential oils to manage pain, enhance the quality of life and promote health and wellness. Studies have shown that plant based aromas have therapeutic properties and treat a variety of systems such as the ability to improve agitation, aggression, influence mood, depression, reduce stress, anxiety and behavior.

Aromatherapy has been in existence for thousands of years using scents and oils for therapeutic purposes. Aromatic plant based compounds were used in ancient medicine. They are the essential oils extracted from plants for use of treating physical and psychological health problems. Aromatherapy helps integrate the mind, body and spirit. It works at a physiological, psychological and molecular level. It can be absorbed on the skin, as a tincture, taken internally or the olfactory system. Essential oils are defined as steam distillates that are obtained from aromatic plants and obtained through petrochemical solvents. They can be complex and made of many different chemical components or molecules. Essentials oils all have a variety of different aromas, medicinal properties and characteristics that make each one unique and diverse. Preclinical research studies that the essential oil bergamot (BEO) fruit, (citrus bergamia) modulates sensitive perception of pain in different models of nociceptive, inflammatory and neuropathic pain modulating endogenous systems. Peppermint oil is an anti-inflammatory used to treat rheumatism and arthritis, fatigue, digestion, nausea and many other ailments.

Existing solutions to poor indoor air quality include a combination of actions such as removing the pollutant sources; increasing ventilation rates and improving air distribution; and cleaning the indoor air.

Urban people spend about $85-90 \%$ of their time indoors (residential and public spaces), which can explain the direct relationship between IAQ and public health risk. The EPA ranked IAQ in the top five public health concerns. Indoor air pollutants (IAQ) such as fine particles, bioaerosols and gaseous compounds appear to be important contributors to poor air quality in domestic and various industrial settings. They have a negative impact on human health causing discomfort as well as acute and chronic diseases. Sick building syndrome (SBS), manifested by ocular, nasal and cutaneous irritations, allergies, respiratory dysfunction, headache, and fatigue is one of the most typical indicators of poor indoor air quality.

Over a period of time upon the inhabitants of these buildings, workers are exposed to the chemicals and reactive by products that adversely affect inhabitants of these buildings that complained about health problems such as itchy eyes, skin rashes, drowsiness, respiratory and sinus congestion, headaches and other allergy symptoms. Based on the airtight sealing of the buildings, trace chemicals, and the synthetic building materials, that are known to emit off gas organic compounds have contributed to disease symptoms and health complaints [2]. Plants, are natural air pollution fighters a which proved effective as natural pollution fighters included those available at most florists or garden centers: Gerbera Daisy, Peace Lily, Bamboo Palm, Spider Plant, Warneckei, Dracaen Marginata, Mass Cane/Corn Cane, Pot mum, Chinese Evergreen, Marginata, Spathophyllum, Janet Craig and English Ivy Chinese Evergreen, Ficus, Weeping Fig, Golden Pothos, Heart Leaf Philodendron, Aloe Vera, Mini-Schefflera, Peperomia, Mother-In Law's Tongue lead the field in effectively reducing levels of a number of noxious gases found in almost every home and office building. Plants have been found effective in reducing the levels of common toxins such as benzene, formaldehyde and trichloroethylene (TCE).

\section{Purpose}

The purpose of the study is to analyze the attributes and effects of plants, aromatherapy and their influence on health and longevity within healthcare environments and to educate communities on how to create holistic, positive and healing environments with plants and aromatherapy. The potential value of the study is to improve quality of life and longevity for individuals within healthcare environments and creating a holistic and green environment.

The purpose of this study is to educate TCM practitioners, healthcare professionals in the community on creating positive environmental and sensory influences, prolonging healthy and longevity on creating less indoor air pollution, incorporating plants and aromatherapy in all indoor environments, creating a healing, healthy and green environment for our TCM Clinics, Hospitals, Healthcare Offices \& Integrative Centers. This research synthesis will be of value to CAM, Integrative Medicine, medical community, Holistic healthcare environments such as hospitals, and our future posterity that will create the green buildings of our future.

\section{Theoretical Assumptions}

Healthcare facilities were traditionally built to just provide the functional delivery of healthcare. Research has shown that the idea of the built healthcare environment having an impact on the health and well being of patients, that more attention is being paid to the psychological consequences of architectural choices [3]. Healing environments are the concept that the physical 
environment of healthcare settings can make a difference in how quickly the patient recovers or adapts to specific or acute and chronic conditions'.

The three dimensions of the physical healthcare environment that can have effects on health and well-being are architectural features (spatial layout, room size, fengshi), ambient feature (lightening, odors) and interior designs (decor, color, artwork, indoor plants).

Research has been shown that restorative environments which expose patients to natural elements, healing properties of nature, in natural settings is an effective way of reducing stress, recovery from illness and diseases in hospital environments. If nature is incorporated and becomes part of the hospital, patients feel more relaxed, calm, and recover quicker from their ailments. Natural elements affect feelings of stress through perceived attractiveness of an environment.

People have innate tendency to gravitate toward natural setting and environments in a built healthcare environment by evolution. People biologically adapt to nature as opposed to built environments. According to one the researchers, Wohlwill [4], stated that nature might be processed more easily and efficiently because the brain and sensory systems evolved in natural environments [4].

Environmental stimuli can facilitate reducing the effects of negative stimuli by adding positive stimuli to the environment by promoting health and well-being. Variables such as indoor plants, colors, and music all help create environments that generate positive feelings. An environmental stimulus changes the environment and influences the patient in a positive way. The environment is very influential on our mood, feelings, and attitude. Just by having plants in the same room and in close proximity, by looking at a view from the window, and/or by the presence of art, the environment can impact health significantly.

Natural elements of bringing nature into hospital are another alternative to a faster recovery and reducing stress in-patient. This study from the Netherlands supported the idea that indoor plants may have beneficial effects on health and well-being, and that the presence of indoor plants in a hospital room leads to a reduction of perceived stress in patients, as well as a perception of beauty, attractiveness and aesthetically pleasing to the eye. It was found that pain control was better for patients exposed to nature. Lohr and Pearson-Mims studied that the presence of indoor plants increased pain tolerance. Participants were placed in a room with plants, and a control room with no plants. The results showed that a significantly larger proportion of respondents in the room with plants had higher pain tolerances. The room with plants showed more of a positive, pleasant, and calming effect than either of the control rooms.

In 2000, Lohr and Peason- Mimsin, researchers that studied if the presence of indoor plants would increase pain tolerance. Participants were either placed in a room with plants or a room with non-plant objects or control room where there were no objects. The results showed that the participants that were exposed to plants were able to keep their hand in the ice water for
5 minutes longer, had an increase in pain tolerance as compared to the other conditions. The room with the plants had more positive, calming effect that the other control rooms. As a result indoor plants have beneficial effects on the health and well-being of the plant.

Aromatherapy is the art and science of utilizing naturally extracted aromatic essences from plants to balance, harmonize the body. There are three different types of aromatherapy used clinical, holistic and aesthetic. Clinical aromatherapy helps relieve stress and anxiety. Holistic aromatherapy improves the overall well-being, focuses on the whole person and improves the quality of life. Aesthetic aromatherapy uses aromatic essences in various tinctures, oils and creams that are primarily for skin care [5]. Martin in 1996, reported decreases in anxiety levels in patients undergoing computerizedaxial-topography (CAT) scans while in the presence of ambient heliotropin, a vanilla-like odor, relative to controls. Similarly, the sedative reputation of lavender has been evidenced in studies of anxiety reduction and mood improvement in a range of situations [6]. He compared the effects of lavender and hiba oil on female patients with chronic haemodialysis and found hiba oil to significantly decrease mean levels of anxiety and depression, where lavender was found only to exert a beneficial effect on anxiety alone. It is worth noting however that no benefit was present in a study investigating the effect of aroma on anxiety in pre-procedural abortion patients. Further to investigations of subjective mood, Brownfield reported that massage with topical administration of lavender resulted in more pronounced analgesic effects than massage alone among patients with chronic rheumatoid arthritis. In addition, to studies of sedative or relaxing aromas, peppermint, jasmine, and rosemary oils have all shown arousing properties in keeping with their collective reputation.

Diego found electroencephalogram (EEG) readings to show increased beta-power following lavender inhalation, implying neurological sedation and corroborating subjective reports of calmness, whereas jasmine has been demonstrated to produce increased alpha-power in the frontal cortices, indicative of increased arousal. Peppermint or (Bohe) Chinese pinyin, is a popular Chinese herb, that appears capable of reliably producing small EEG and electromyogram (EMG) or muscular-conductance fluctuations during REM and NREM sleep [7], a finding that is able to rule out the possible effects of expectancy.

Degel \& Koster [8] found inhalation of lavender to improve letter counting and mathematical tasks, relative to inhalation of jasmine, whereas both extracts impaired creativity performance relative to controls. Furthermore, Degel et al. [8] described a beneficial effect of lavender and orange aromas on a measure of implicit memory. Warm reported both peppermint and muguet essences and increased performance on a sustained visual attention task and explored the psychological component of performance effects and ventured interlinked correlations between subjective evaluation of substances and corresponding expectations, relative to task performance. Their results clearly assign a high psychological component to the effects of essential oil aromas. There is a complex pattern of relationships between induced expectancies and aroma effects when investigating the influence of chamomile aroma on cognition and mood. 
Recent assessment of the effect of ylang-ylang aroma on physiological parameters and subjective state demonstrated a possible "harmonization" effect illustrated through reductions in blood pressure and heart rate accompanied by increases in alertness and attentiveness [9]. However, no assessment of cognitive functioning was studied. The accrued evidence therefore supports the proposal of substance-specific effects on subjective state, physiological measures, and to some extent cognitive performance. This study was successful and assessed the impact of peppermint (Mentha piperita), and ylang ylang (Canananga odorata) essential oil aromas on a wide range of cognitive performance measures and improved mood elevation.

Ylang-ylang is widely regarded as possessing sedative and calmative properties and is commonly found in products aimed at aiding relaxation Tisserand. Such a proposition was supported by the increase in "calmness" reported here by participants in the ylang-ylang condition compared to those in both the control and peppermint conditions. The possible stimulating effect of peppermint aroma was only partially supported. A significant difference in the alertness mood dimension was isolated between the two aroma conditions, with peppermint producing a small increase and ylang-ylang a decrease. Peppermint produced a significant improvement in overall quality of memory, compared to both control and ylang-ylang conditions. This factor derives from the accuracy scores from all the tasks of long term and working memory and the improvement versus controls only existed when all these tasks were considered together in this factor.

If the secondary (long term) and working memory subfactors are considered separately, peppermint was only found to be significantly enhancing performance then compared to ylang-ylang. These differences appear to be a reflection of the combination of the enhancement and impairing properties of the two aromas, respectively. Interestingly, the improvement in accuracy of memory for peppermint was not at the cost of speed. The speed of memory factor indicated that ylang-ylang slowed reaction times significantly compared to controls. Peppermint, however, produced no significant change in this factor. The factors derived from the tests of attention revealed a similar pattern of results. For the speed of attention factor, ylang-ylang produced the slowest reaction times, significantly so when compared to peppermint that produced the quickest. Peppermint has also been demonstrated to enhance performance on a range of physical exercise tasks. The authors propose that the effects are due to the aroma producing a change in an unidentified dimension of mood and consequently in the level of motivation of the participants. Although a small increase in alertness was recorded for peppermint aroma in the current study, this was not significant compared to controls.

The direct pharmacological action would require the absorption of volatile compounds into the blood and their subsequent activity at a neuronal level. Although the level of active compounds that may be absorbed from an ambient aroma through the lungs and nasal mucosa is low when compared to other modes of administration; monoterpene components of rosemary have been detected in the blood of rodents exposed to the vapors of this essential oil attractive aspect of a pharmacological mechanism for the affect of aromas on cognition is the concept of substancespecificity.

Each aroma delivering a unique pattern of influence on the cognitive factors described. In support of the pharmacological influence of plant-based compounds research has provided evidence that is pertinent here. Wake et al. [10] demonstrated that sage possessed neuropharmacological activity, specifically, on the nicotinic and muscarinic acetylcholine systems in homogenate preparations of human cortical cell membranes. There is a longestablished link between the cholinergic system and memory, and it may be the case that other plants such as peppermint and rosemary also possess such activity.

Recent work with animals also supports the proposal that volatile organic plant constituents can have direct pharmacological effects on behavior and physiology. Compounds that emanate from the leaves of certain deciduous trees such as the oak have been collectively termed "green odor" and this has been shown to attenuate stress-induced activation of the hypothalamopituitaryadrenal (HPA) axis in rats [11]. Activation results in persistent hyperthermia, significantly diminished by inhalation of green odor. Lavender aroma was found to produce decrements in performance on secondary memory, speed of memory, and speed of attention compared to controls. In contrast, rosemary only significantly enhanced secondary memory performance. Rosemary increased alertness, creativity and mood compared to lavender and controls, and lavender and rosemary both increased contentedness compared to controls.

These effects bear similarities to, but importantly also distinct differences to the patterns observed here. As a result, each essential oil may possess its own idiosyncratic pattern of influence. This would be consistent with the aroma therapist's view and may be a result of the proportions and structure of the constituent volatile compounds. The data presented here and evidence from elsewhere it seems plausible that the effects observed in studies of the aromas of essential oils maybe a result of a combination of pharmacological, cognitive, and emotional effects. Recent studies have shown that the use of biofeedback and aromatherapy can alter electroencephalographic (EEG) and electrocardiographic (ECG) activity [12]. There were an equal number of male and female subjects that had one out of three conditions biofeedback, sedative aromatherapy that had diffused lavender essential oil or aromatherapy that had diffused jasmine essential oil. The study examined the physiological effects of biofeedback after a twoweek treatment regimen to objectively assess stress reduction. The Fast Fourier transformations (FFT) of the raw EED were used by the mean frequency of EEG and alpha 8-13 Hz activity and power. The participants had four sessions, two sessions per week for two weeks. EEG activity was recorded from the occipital lobe and ECG activity was acquired with electrodes placed on the right wrist and inner ankles. The preliminary results showed that the biofeedback and sedative aromatherapy had an increase in the overall alpha and mean EEG activity, which further concluded reducing overall stress [12] 


\section{Research Hypothesis}

I. I hypothesize that plants and aromatherapy within healthcare environments do influence health and longevity.

II. I hypothesize that plants and aromatherapy within healthcare environments affect the indicators of personal health such as a person's overall mood, heart rate, vital lung capacity and blood pressure.

\section{Importance of the study}

Americans spend nearly $90 \%$ of their time indoors. Poor Indoor Air Quality (IAQ) affects twenty- five percent of US residents either in the workplace or at home. The US Environmental Protection Agency (EPA) ranks poor IAQ among its largest national environmental threats. Its counterpart, the European Environmental Agency (EEA) has pointed to IAQ as one of the priority concerns in children's health and similar issues are, being faced worldwide.

The office equipment and furniture in the buildings are also a contributing factor that is created in the types of materials used in their manufacture and design. All these factors contribute in some of the buildings contain such high levels of contaminants that they are qualified as "sick" because exposure to them results in multiple sickness symptoms such as headache, fatigue, skin and eye irritations, or respiratory illness commonly described as the "sick-building syndrome" (SBS).

It is not natural or normal for people to live in confined, poorly ventilated area for long periods of time. Airplanes are confined places, where there are a large number of people present more indoor air pollution exposed and less oxygen present. Since the late 1970 's, energy has always been scarce and a limited resource. There is an overuse of gas, heating and cooling systems that have created energy efficient buildings and decreasing energy costs. These two designs changes improved energy efficiency by using super insulation and reduced fresh air exchange.

Indoor air contamination is a complex problem involving particles (such as dust and smoke), biological agents (molds, spores), radon, asbestos, and gaseous contaminants such as $\mathrm{CO}$ $\mathrm{CO}_{2}, \mathrm{NOx}, \mathrm{SOx}$, aldehydes and VOCs (Volatile Organic Compounds). VOC are in building pollutants are materials, that are in furniture, cleaning products, mold development, etc. As a result, humans suffer from health problems such as: have irritants in their eyes and/or respiratory tissues, allergies, cancers, liver problems, immunity and nervous conditions and reproductive issues. These irritations are caused by dust, air mists, pollen, cigarette smoke, viruses, activity, and decaying buildings. These particles are very small liquid or solid substances that are in suspension in outdoor air, exhaust from combustion, and carpets. There is an indirect effect through the biological production of toxins, bacteria, molds, and gases such as $\mathrm{CO}, \mathrm{CO}_{2}, \mathrm{NOx}$, and formaldehyde.

Formaldehyde, one of the most highly reported symptoms from exposure of this chemical is found in virtually all indoor environments, including foam insulation, particle board or pressed wood products. It is used in consumer paper products treated with resins, including grocery bags, waxed papers, facial tissues and paper towels. Most household cleaning agents contain formaldehyde. The EPA recently has conducted research, which indicates that formaldehyde, which is an irritant to mucous membranes of the eyes, nose and throat, causes a rare type of throat cancer in long-term occupants of mobile homes. It is highly reactive chemical that combines with allergic contact dermatitis [13]. Evidence-based design is evidence-based medicine supporting healthcare environments that promote health and well-being. There have been studies in the past that have shown the use of evidence-based design impacting health-related outcomes of length of stay, pain medication intake, stress and mood. Understanding physical environment stimuli in healthcare facilities will automatically create positive environments that affect the healing process and well-being of patients.

In 1999, Ulrich stated that stress is a major problem in health care facilities. In addition to ill health, which may promote stress; effective care environments are often psychologically stressful [1]. Stress itself leads to negative health outcomes and may hinder recovery processes. Ulrich [1] suggested that environments with conductive characteristics could alleviate stress experiences.

According to his conceptual model, the key features for individuals in supportive, health promoting environments are sense of control, access to privacy, access to social support, possibilities for movement and exercise and access to nature and to other positive distractions. These characteristics of an environment facilitate an individual's abilities to recover and cope with stress, which in turn results in improved health.

The architectural features and dimensions are permanent aspects of the physical healthcare environment such as hospital size and shape of the room, layout of the hospital, placement of windows and light. Interior design features are similar to accessories, creativity, such as colors, interior plants, furnishings, and art work.

There is research on restorative environments that certain and specific types of environments have the capability of promoting stress that natural settings have restorative effects [14].

Trees and the open green spaces have multiple uses and their presence in the outdoors make a major contribution to the saving of energy inside of buildings, and the improvement of the microclimate in the urban spaces adjacent to buildings and in the urban sub areas. The attributes of green urban spaces that affect the urban microclimate positively are as follows: high rate of absorption of solar radiation, low heat capacity and thermal conductivity compared to the structural, the materials of buildings and urban open spaces, the reduction of air temperature and transpiration, the decreased infrared radiation, the reduction of wind speed, dust and pollutants from the air; and the presence of the trees provide sound protection and protective coverage [1529].

This study focuses on plants and their effects on influencing the health and longevity of all persons who work, are treated, and/or are providing treatment within a healthcare facility. The optimal 
healing and therapeutic space include patients, employees and other healthcare practitioners, achieving better quality of life and promoting health and longevity. It is the intention of this study to analyze and identify plants and their use within the healthcare environment and how they contribute to the overall health and longevity of individuals within those environments. This researcher will also identify and analyze the elements, which comprise a healing environment such as of plants, use of aromatherapy and access to sunlight and apply that knowledge to the development of a healing healthcare environment. Perhaps this study can contribute to the overall education of people at large about creating therapeutic living environments for themselves as well.

\section{Scope of the Study}

\section{Data Collection, analysis and reporting}

Research Synthesis data will be complied through online search of medical journals through UCLA Catalog, Pubmed, Google Scholar, Science Direct, Web of Science, CINAHL, Biosis. Published books, journal articles.

Inclusion criteria for Literature Review/Research Synthesis and articles from 1991 to present human studies will be included, Plant Studies, Case studies done with Plants Articles from all countries will be accepted, peer articles used

Exclusion criteria for Research Synthesis: no articles older than 1984 and no non- peer review articles. Data will be collected and analyzed using article abstraction form. This data will be complied to further study humans and the plants and put into capstone dissertation.

This research synthesis will be very valuable to the world, TCM. Community, TCM clinics, Integrative Medicine Clinics, patients and the future of healthcare environments, natural green medicine and integrative medicine (collaboration of western and eastern medicine). It will inspire healthcare practitioners and the community to create alternative green medicine and green hospitals and healthcare environments.

The value of this study is to educate the medical and nonmedical community on creating positive, healing, healthy and holistic green environment, with plants, aromatherapy, used in indoor environments.

\section{References}

1. Ulrich R, Simons (1991) Stress recovery during exposure to natural and urban environments. Journal of environmental psychology 11: 201-230.

2. Ruga W (1989) Designing for the sixth sense. Journal of Health Care Interior Design 1: 29-34.

3. Dijkstra K, Pieterse ME, Pruyn A (2008) Stress-reducing effects of indoor plants in the built healthcare environment: The mediating role of perceived attractiveness. Prev Med 47(3): 279-283.

4. Wohlwill WF (1983) The concept of nature: a psychologist's view. In: Altman I \& Wohlwill F (Eds.), Human Behavior and the Environment. $\left(6^{\text {th }}\right.$ edn), Behavior and the Natural Environment, Plenum, New York, USA, p. 5-37.
5. Metcalfe J (1989) Herbs and aromatherapy. Exter, Webb and Bower, UK.

6. Buchbauer G, Jirovetz L, Jäger W, Dietrich H, Plank C (1998) Aromatherapy: Evidence for the sedative effect of the essential oil of lavender after inhalation. Z Naturforsch C 46(11-12): 1067-1072.

7. Badia P, Wesensten N, Lammers W, Culpepper J, Harsh J (1990) Responsiveness to olfactory stimulus presented in sleep. Physio Behav 48(1): 87-90.

8. Degel J, Köster EP (1999) Odours: Implicit memory and performance effects. Chem Senses 24(3): 317-325.

9. Hongratanaworakit T, Buchbauer G (2004) Evaluation of the harmonizing effectof ylang-ylang oil on humans after inhalation. Planta Med 70(7): 632-636.

10. Wilson MA (2000) Social attributions based on domestic interiors. Journal of Environmental Psychology 20: 343-354.

11. Akutsu H, Kikusui T, Takeuchi Y, Sano K, Hatanaka A, et al. (2002) Alleviating effects of plant derived fragrances on stress-induced hyperthermia in rats. Physiol Behav (75): 355-360.

12. Ceballos, Rachel M, Matthews, Kelli, Catledge, et al. (2000) Quantifying States of Relaxing with EEG and ECG, A comparison of biofeedback and aromatherapy. Psychophysiology 37(1): 232.

13. Gammage RB, Kaye SV (1984) Indoor Air and Human Health. Proceedings of the Seventh Life Sciences Symposium. Lewis Publishers, Inc. UK, p. 29-31.

14. Hartig T, Böök A, Garvill J, Olsson T, Gärling T (1996) Environmental influences on psychological restoration. Scand J Psychol 37(4): 378393.

15. Degel J, Piper D, Köster EP (2001) Implicit learning and implicit memory for odors: The influence of odor identification and retention time. Chem Senses 26(3): 267-280.

16. Wilson MA (2000) Social attributions based on domestic interiors. Journal of Environmental Psychology 20: 343-354.

17. Wargocki P, Wyon DP, Sundell J, Clausen G, Fanger PO (2000) The effects of outdoor air supply rate in an office on perceived air quality, sick building syndrome (SBS) symptoms and productivity. Indoor Air 10(4): 222-236.

18. Pilon-Smit (2005) Phytoremediation. Annu Rev Plant Biol 56: 15-39

19. Wood RA, Burchett MD, Alquezar R, Orwell RL, Tarran J, et al. (2006) The potted- plant microcosm substantially reduces indoor air VOC pollution: I. Office field study. Water Air Soil Pollut 175(1-4): 163180

20. Ilmberger J, Heuberger E, Mahrhofer C, Dessovic H, Kowarik D, et al (2001) The influence of essential oils on human attention. Chemical Senses 6(3): 239-245.

21. Georgiou K (1999) Flora and vegetation: plants and attributes as for their use for the improvement of built environment Introduction in natural and human environment. Hellenic Open University, Patra, India, pp. 313

22. Frey PW, Colliver JA (1973) Sensitivity and response measures for discrimination learning. Learning Motivation 4: 327-342.

23. Dimoudi AM, Nikolopoulou (2003) Vegetation in the urban environment: microclimatic analysis and benefits. Energy and Buildings 5: 9-76. 
24. Grahn P (1991) Om parker's betydelse. Stad \& Land nr. 93. Alnarp, Swedish University of Agricultural Sciences, Institute for Landscape Planning. Sweden.

25. Buckle J (1999) Use of aromatherapy as a complementary treatment for chronic pain. Altern Ther Health Med 5(5): 42-51.

26. Campenni CE, Crawley EJ, Meier ME (2004) Role of suggestion in odor induced mood change. Psychol Rep 94 (32): 1127-1136.

27. Alagappan G, Cowan R (2003) Substrate inhibition kinetics for toluene and benzene degrading pure cultures and a method for collection and analysis of respirometric data for strongly inhibited cultures. Biotechnology Bio eng83(7): 798-781.

28. Aydogan LD, Montoya (2011) Formaldehyde removal by common indoor plant species and various growing media. Atmos Environ (45): 2675-2682.

29. Aguado S, Polo AC, Bernal MP, Coronas J, Santamaria J (2004) Removal of pollutants from indoor air using zeolite membranes. J Member Sci (240): 159-166. 